\title{
Free estradiol index levels associated with high sensitivity CRP levels in male children
}

\author{
Mehmet Ağırbaşl11 ${ }^{1}$ Azra Tanrıkulu², Munir Azizy ${ }^{3}$ \\ ${ }^{1}$ Department of Cardiology, Medeniyet University School of Medicine; ${ }^{2}$ Department of Cardiology, Maltepe State Hospital; \\ ${ }^{3}$ Department of Internal Medicine, Marmara University School of Medicine, Istanbul, Turkey. E-mail: magirbasli@gmail.com \\ Received: 17 November 2016, Accepted: 24 January 2017
}

SUMMARY: Ağırbaşlı M, Tanrıkulu A, Azizy M. Free estradiol index levels associated with high sensitivitiy CRP levels in male children. Turk J Pediatr 2017; 59: 49-55.

Biomarkers of inflammation such as high-sensitivity C-reactive protein (hsCRP) associate with subclinical atherosclerosis. Atherosclerosis is an early onset disease in life. Sex hormones and puberty modulate metabolism in children. Studies indicate that low sex hormone binding globulin (SHBG) levels associate with insulin resistance and metabolic syndrome in children. The aim of this study is to study the correlation between sex hormones and hs-CRP levels in children and adolescents. The study sample was derived from a cross sectional survey on the prevalence of cardiovascular risk factors in a representative sample of school children (8-17 year-old) in Istanbul, Turkey. In addition to anthropometric and biochemical characteristics of cardiovascular risk, sex hormones such as free androgen index, free estradiol index (FEI), SHBG and hs-CRP levels were measured in all study participants: 91 boys (12.4 \pm 3.4 years) and 77 girls $(12.7 \pm 3.4$ years) were included in the study. Median (interquartile range) hs-CRP levels were similar among boys and girls [0.36 $(0.9)$ versus $0.45(0.7) \mathrm{mg} / \mathrm{dl}, \mathrm{p}=0.725]$. Gender stratified analysis displayed that hs-CRP levels positively correlated with FEI levels $(r=0.438, p<0.001)$ in boys. Linear regression analysis was performed to determine the predictors of hs-CRP. Among covariates of FEI, homeostasis model assessment-estimated insulin resistance, body mass index, age, and SHBG; FEI was shown to significantly and independently predict hs-CRP levels in boys $[\beta=2.758, \mathrm{p}<0.001,95 \%$ confidence interval (CI) for $\beta 1.471-4.045]$. FEI levels associate with subclinical inflammation in boys. Future studies may elucidate the role of sex hormone levels in inflammation among children.

Key words: cardiovascular risk, children, C-reactive protein, sex hormones.

Atherosclerosis is an early onset process beginning in childhood ${ }^{1}$. Since cardiovascular risk is multifactorial with environmental, vascular, endocrine and genetic components, successful prevention of atherosclerosis requires recognition and delineation of the precipitating causes. Current endemic of childhood obesity displays that insulin resistance and metabolic syndrome start early in life. Puberty and hormonal changes are components of maturation process in children. Recent study indicates that low sex hormone binding globin (SHBG) levels associate with insulin resistance and metabolic syndrome in children ${ }^{2}$. Metabolic syndrome and cardiovascular risk initiate a low grade inflammation in the onset of atherosclerosis ${ }^{3}$. Chronic low grade inflammation plays an important role in the development of cardiovascular disease and C-reactive protein (CRP) is a biomarker for systemic inflammation ${ }^{3}$. CRP can be measured by high sensitivity methods (hs-CRP). High sensitivity CRP levels are used in risk stratification of patients for cardiovascular events ${ }^{3}$. However, the time of onset for the association between cardiovascular risk and low grade inflammation throughout the lifespan remains unclear. Cardiometabolic risk factors in children, such as TC-to-HDL-C (total cholesterol to high density lipoprotein cholesterol) ratio correlate with hs-CRP levels ${ }^{4}$. Since, atherosclerosis has its origins in childhood ${ }^{5}$, the correlation 
between sex hormone levels and hs-CRP is of interest. In this study, we analyze the relation between sex hormones and hs-CRP levels among children and adolescents. The aim is to test the hypothesis that androgen/estrogen balance may contribute to the changes in hsCRP levels among children and adolescents.

\section{Material and Methods}

\section{Study population}

The study was conducted among school students living in an urban, low to middle socio-economic environment in Istanbul, Turkey. The study sample was derived from a cross sectional survey on the prevalence of cardiovascular risk factors in a representative sample of school children in Istanbul, Turkey. Five different state elementary and secondary

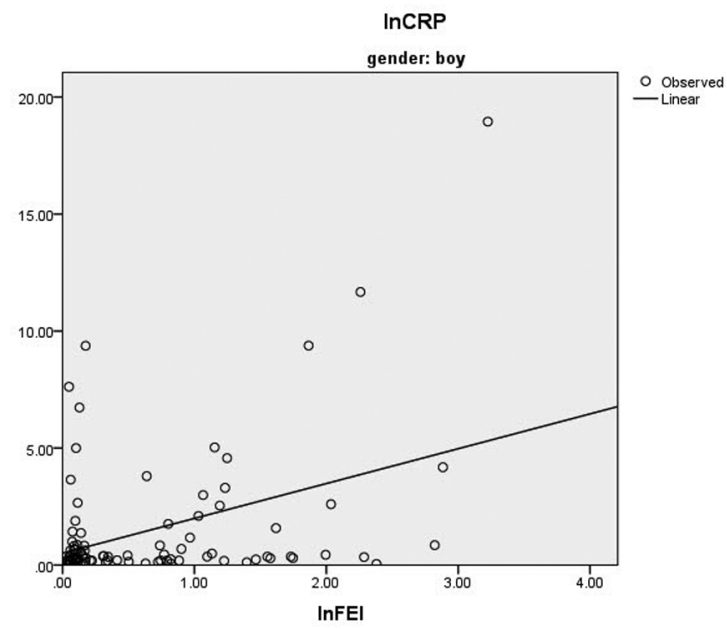

Fig. 1. The regression curve between ln FEI (free estradiol index) and ln hs-CRP $(\mathrm{mg} / \mathrm{dl})$ in boys $\left(\mathrm{R}^{2}=0.16, \mathrm{p}<\right.$ 0.001). schools were selected. Students who did not want to participate in the survey were excluded (around 10\% of the elementary school children and $20 \%$ of the secondary school children). One hundred and sixty-eight children and adolescents aged 8-17 years were included in the study.

\section{Data collection}

Children were instructed to fast for at least 12 hours before the venipuncture. Their compliance was ascertained by an interview on the day of examination. The fasting status was based on self-report. For biochemistry, hs$\mathrm{CRP}$ and sex hormone assays; samples were kept at $2-8^{\circ} \mathrm{C}$ until the analysis was performed the same day in the internationally accredited laboratory. Blood samples were drawn between 8-9 am and screening took place at the student health offices of the schools during regular school hours. Adequate space is provided by the school authorities within the school premises for the study.

The study was approved by the Institutional Ethics Committee. Subjects were given case numbers and identities were kept confidential. Parents of the school children were informed. At least one parent signed an informed consent for the participation after having read an explanatory note. Family history of coronary artery disease was defined as having a first or second degree relative with established coronary artery disease.

\section{Anthropometric measurements}

Weight, height, biceps skin fold thickness, waist and hip circumference and arm span were measured by formally trained physicians. The

Table I. Demographics, Anthropometry and Biochemical Characteristics of the Study Participants.

\begin{tabular}{llll}
\hline Characteristics & $\begin{array}{l}\text { Boys } \\
(\mathrm{n}=91)\end{array}$ & $\begin{array}{l}\text { Girls } \\
(\mathrm{n}=77)\end{array}$ & $\mathrm{p}$ \\
\hline Age (years) & $12.4 \pm 3.4$ & $12.7 \pm 3.4$ & 0.573 \\
Overweight or obesity based on BMI, n (\%) & $15(16.5)$ & $12(15.6)$ & 0.875 \\
Waist circumference $>90^{\text {th }}$ percentile, $\mathrm{n}(\%)$ & $9(9.9)$ & $14(18.2)$ & 0.120 \\
Blood pressure $>95^{\text {th }}$ percentile, $\mathrm{n}(\%)$ & $23(25.3)$ & $19(24.7)$ & 0.929 \\
Body mass index $\left(\mathrm{kg} / \mathrm{m}^{2}\right)$ & $19.0 \pm 4.0$ & $19.4 \pm 4.2$ & 0.508 \\
Skin fold thickness $(\mathrm{mm})$ & $8.0(6.0)$ & $12.0(5.0)$ & $<0.001$ \\
C-reactive protein $(\mathrm{mg} / \mathrm{dl})$ & $0.36(0.90)$ & $0.45(0.72)$ & 0.725 \\
\hline
\end{tabular}

Median (interquartile range) is displayed for variables with skewed distribution.

Mean \pm standard deviation is displayed for variables with normal distribution. 
Table II. Distribution of Sex Hormone Levels in Children

\begin{tabular}{llll}
\hline Sex Hormone Levels & $\begin{array}{l}\text { Boys } \\
(\mathrm{n}=91)\end{array}$ & $\begin{array}{l}\text { Girls } \\
(\mathrm{n}=77)\end{array}$ & $\mathrm{p}$ \\
\hline Free estradiol index & $0.3(1)$ & $0.7(2)$ & 0.001 \\
Free androgen index & $1.0(49)$ & $1.75(4)$ & 0.712 \\
Sex hormone-binding globulin (nmol/L) & $73 \pm 52$ & $69 \pm 32$ & 0.533 \\
Testosterone (ng/ml) & $0.3(4.3)$ & $0.3(0.5)$ & 0.573 \\
Estradiol $(\mathrm{pg} / \mathrm{ml})$ & $11.1(16)$ & $33.7(70)$ & $<0.001$ \\
\hline
\end{tabular}

Median (interquartile range) is displayed for variables with skewed distribution.

Mean \pm standard deviation is displayed for variables with normal distribution.

weight of the children wearing minimal clothing was measured to the nearest $0.1 \mathrm{~kg}$ with a portable electronic scale. Height was measured with a fiberglass tape. Body mass index (BMI) was calculated as weight $(\mathrm{kg}) /$ height (meter) square. Body circumferences were measured with subjects in a standing position. Hip and waist (just above the iliac crest) circumferences and arm span were measured to the nearest $0.1 \mathrm{~cm}$. Based on a nationally representative sample of Turkish Children and Adolescents, we determined the subjects with increased waist circumference ( $>90$ th percentile) ${ }^{6}$. Biceps skin fold thickness was measured to the nearest 1.0 $\mathrm{mm}$ with a Holtain caliper. Age- and sex-specific cut-off points of BMI were used to assess the overweight and obesity status ${ }^{7}$.

\section{Sex hormone and hs-CRP levels}

Testosterone, estradiol (E2) and SHBG levels were measured by chemiluminescence immunoassay method using Modular E170 kit (Roche ${ }^{\circledR}$ Diagnostic). The plasma level of hs-CRP was measured with the autoanalyser (Aeroset, Abbott ${ }^{\circledR}$ ) by spectrophotometric commercial kit (Scil Diagnostics GmbH,
Germany). To estimate free (non-protein bound) testosterone, we calculated the free androgen index (FAI), the molar ratio of total testosterone to $\mathrm{SHBG}^{8}$. To convert testosterone to nanomolar, the nanogram per deciliter value is multiplied by 0.0347 . FAI is calculated according to the following equation: FAI = $100 \times$ total testosterone/ SHBG. Likewise, to estimate the free E2 concentration, the free estradiol index (FEI), the molar ratio of E2 to SHBG was calculated ${ }^{8}$. To convert E2 to picomolar, the picogram per milliliter value is multiplied by 3.67. FEI is calculated by the ratio of E2 to SHBG multiplied by 100 .

Insulin resistance was calculated according to the homeostasis model assessment formula. Homeostasis model assessment-estimated insulin resistance (HOMA-IR) is calculated using the formula (glucose $(\mathrm{mmol} / \mathrm{L}) \mathrm{x}$ fasting insulin $(\mu \mathrm{U} / \mathrm{ml}) / 22.5)$. HOMA-IR has been validated as a measure of insulin resistance in non-diabetic children ${ }^{9}$.

Analyses were performed in an accredited laboratory (Centro Laboratories which are based in Istanbul/Turkey). The laboratory is accredited

Table III. Partial Correlation Analysis After Controlling for Age Between Sex Hormone Levels and ln hs-CRP in Children and Adolescents.

\begin{tabular}{|c|c|c|c|c|c|c|}
\hline \multirow[b]{2}{*}{ Sex Hormone } & \multicolumn{2}{|l|}{ Boys } & \multicolumn{2}{|l|}{ Girls } & \multicolumn{2}{|c|}{ All subjects } \\
\hline & $\mathrm{r}$ & $\mathrm{p}$ & $\mathrm{r}$ & $\mathrm{p}$ & $\mathrm{r}$ & $\mathrm{p}$ \\
\hline $\ln \mathrm{FEI}$ & 0.438 & $<0.001$ & -0.055 & 0.634 & 0.009 & 0.913 \\
\hline $\ln$ FAI & 0.123 & 0.246 & 0.111 & 0.338 & 0.154 & 0.046 \\
\hline SHBG (nmol/L) & -0.069 & 0.517 & -0.050 & 0.666 & -0.092 & 0.238 \\
\hline ln estradiol $(\mathrm{pg} / \mathrm{ml})$ & 0.124 & 0.245 & -0.069 & 0.552 & -0.045 & 0.567 \\
\hline ln testosterone $(\mathrm{ng} / \mathrm{ml})$ & -0.002 & 0.982 & 0.118 & 0.311 & 0.101 & 0.192 \\
\hline
\end{tabular}

FEI: free estradiol index; FAI: free androgen index; SHBG: sex hormone-binding globulin; hs-CRP: high-sensitivity C-reactive protein

Because of the skewed distribution of concentrations of testosterone, FAI, estradiol, FEI, and hs-CRP; these variables were log-transformed $(\ln )$ for correlation analysis. 
Table IV. Linear Regression to Find the Predictors of ln-CRP in Children and Adolescents

\begin{tabular}{lllllll}
\hline & For boys & \multicolumn{5}{c}{ For girls } \\
\hline Predictors & $\beta$ & $\mathrm{p}$ & $95 \%$ CI for $\beta$ & $\beta$ & $\mathrm{p}$ & $95 \%$ CI for $\beta$ \\
Age & -0.198 & 0.129 & -0.455 to 0.059 & -0.426 & 0.099 & -0.934 to 0.083 \\
BMI & -0.068 & 0.539 & -0.286 to 0.150 & 0.347 & 0.030 & 0.034 to 0.659 \\
SHBG & 0.010 & 0.272 & -0.008 to 0.028 & 0.020 & 0.375 & -0.025 to 0.065 \\
ln HOMA-IR & 0.403 & 0.136 & -0.130 to 0.936 & 0.047 & 0.897 & -0.639 to 0.733 \\
ln FEI & 2.758 & $<0.001$ & 1.471 to 4.045 & 1.049 & 0.663 & -3.723 to 5.821 \\
\hline
\end{tabular}

FEI: free estradiol index; FAI: free androgen index; HOMA-IR: homeostasis model assessment-estimated IR; BMI: body mass index; HDL-C: high density lipoprotein cholesterol; TG: triglyceride; CI: confidence interval; CRP: C-reactive protein Because of the skewed distribution of concentrations of testosterone, FAI, estradiol, FEI, and hs-CRP; these variables were log-transformed (ln) for multiple linear regression.

by DAR (Deutscher Akkreditierungs Rat) according to ISO 15189 by clinical laboratory accreditation committee. Measurement uncertainty of each method in use for this laboratory are $5.07 \%$ for insulin, $3.37 \%$ for testosterone, $3.88 \%$ for E2 and $5.78 \%$ for SHBG.

\section{Statistical analysis}

Chi-square test was used for categorical variables. The one-sample Kolmogorov Smirnov test was used to verify the normality of data distributions. Mean \pm standard deviations (SD) are displayed for variables with normal distribution. Median and interquartile range (IQR) are provided for variables with skewed distribution (E2, testosterone, FEI, FAI, and hs-CRP). Statistical comparisons of quantitative data were performed by a student t-test or Mann-Whitney U-test. After controlling for age, partial correlation analysis is performed between the sex hormones and hs-CRP. Multiple linear regression analysis is performed to find the predictors of hs-CRP. Because of the skewed distributions; testosterone, FAI, E2, FEI, and hs-CRP are log-transformed (ln) for regression and correlation analysis. Variables entered into the regression model are SHBG, BMI, age, In HOMA-IR, ln FEI (boys) and ln FAI (girls). A value of $p<0.05$ on the twosided test is considered statistically significant. Statistical analyses are performed using SPSS13 for Windows (SPSS Inc., Chicago, Ill., Nr. 9026510).

\section{Results}

Demographic characteristics of the study participants and hs-CRP levels were shown in Table I. A total of 168 children and adolescents were enrolled in the study (91 boys and 77 girls). Overweight or obesity were observed in 27 subjects [15 boys $(16.5 \%)$ versus 12 girls (15.6\%), $\mathrm{p}=0.875]$. Median (IQR) hs-CRP levels were similar among boys and girls [0.36 (0.90) versus $0.45(0.72) \mathrm{mg} / \mathrm{dl}$, $\mathrm{p}=0.725]$. The distribution of sex hormone in study participants were displayed in Table II.

\section{Sex hormone levels and hs-CRP}

Table III displayed the correlation analysis between sex hormone and hs-CRP levels in children and adolescents. FEI levels significantly correlated with hs-CRP levels in boys. There was no significant correlation between sex hormones and hs-CRP levels in girls. Linear regression analysis was performed to determine the predictors of ln hs-CRP. Among covariates of SHBG, HOMA-IR, BMI, age, ln FEI (boys), and ln FAI (girls), ln FEI was shown to significantly and independently affect hs-CRP levels in boys (Table IV).

The regression curve between $\ln$ FEI and $\ln$ hs-CRP in boys were displayed in Figure 1 $\left(\mathrm{R}^{2}=0.16, \mathrm{p}<0.001\right)$.

\section{Discussion}

Clinical studies indicate that atherosclerosis and increased cardiovascular risk initiate low grade inflammation. Sex hormones and puberty modulate cardiovascular risk in children. Low SHBG levels (as usually observed in obese children) associate with insulin resistance and metabolic syndrome in children ${ }^{2}$. Novel inflammatory biomarkers associate with cardiovascular risk factors in obese children ${ }^{10}$. Risk factors such as hyperinsulinemia, obesity, hypertension and hyperlipidemia, are common among obese children ${ }^{5,11}$. In a study of 354 
children, obese children display higher levels of hs-CRP, interleukin and tumor necrosis factor- $\alpha$ compared to the non-obese subjects ${ }^{10}$. Obese children with family history of cardiovascular disease display higher hs-CRP levels compared to those without family history of cardiovascular disease ${ }^{12}$.

The current knowledge is limited on the distribution of sex hormones in children and adolescents, particularly, their relation to inflammation. Previous observations indicate that sex hormone levels and androgen/ estrogen balance play an important role in the onset of metabolic syndrome in children and adolescents ${ }^{13}$. Therefore, the correlation between sex hormone levels and hs-CRP is of interest in children. In this study, we analyzed the relation between sex hormones and hsCRP levels in children living in an urban, low to middle socio-economic environment. The hypothesis is that androgen/estrogen balance contributes to the subclinical inflammation in children and adolescents. FEI levels significantly and positively correlate with hs-CRP levels in male children and adolescents. Cause and effect relationship between the sex hormone and hs-CRP levels remains to be elucidated. Estrogens are not exclusive to the female gender and occur in moderate circulating levels in men ${ }^{14}$. Puberty and aging in children are associated with alterations in a number of cardiovascular risk factors including adiposity, insulin resistance, and obesity ${ }^{15}$. Coupling of the low grade inflammation with cardiovascular risk is crucial in understanding the onset of atherosclerosis ${ }^{16}$. In addition to the wide range of general physical development and changes in children and adolescents, it is imperative to address age-related affective alterations arising in testosterone/estradiol imbalance possibly as a cause of cardiovascular risk and low grade inflammation ${ }^{17}$. Although $75-90 \%$ of E2 in male gender results from aromatisation of testosterone in the steroid pathway, E2 can counterbalance with testosterone for its physiological effects ${ }^{18}$. For instance in elderly men, E2 has many opposing physiological functions and the progressive testosterone decline is associated with relative and/or absolute increase in serum E2 ${ }^{19,20}$. Cardiovascular risk increase in elderly men is concurrent with E2-testosterone imbalance ${ }^{19,20}$. Thus, it is likely that the relationship of FEI and cardiovascular risk in men is bidirectional throughout the life span; high FEI levels associate with low grade inflammation in children and enhanced cardiovascular risk elderly men. Thus, relative and/or absolute increase in FEI is a biomarker of cardiovascular disease in elderly men ${ }^{19}, 20$.

Gender related differences are observed in the relation between sex hormone and hs-CRP levels in children and adolescents. Similarly, ethnic differences occur in the distribution of hs-CRP and sex hormones. For instance, hs-CRP values in Turkish men and women are 15 to $25 \%$ higher than those given for American or European counterparts ${ }^{21}$. Hergenc et al. ${ }^{22}$ report that compared with Germans, Turkish middle-aged adults had similar total testosterone but lower SHBG levels. Cardiovascular risk factors such as obesity lower SHBG levels and lead to higher FEI, insulin resistance and inflammation ${ }^{10,11}$.

Our findings are consistent with the published reports 23,24 in adult population. SHBG and testosterone negatively correlate with CRP, whereas estrogen positively correlates with CRP. Similarly, a study of 809 adult men in the USA $^{25}$ reveals that testosterone levels negatively associate with CRP; yet total and free E2 positively associate with CRP and white blood cell levels. Several studies repeatedly report that increased E2 levels in middle-age men indicate worse cardiovascular risk phenotype such as atherogenic lipid profile ${ }^{25}$, coronary artery disease ${ }^{26}$, and increased carotid artery intima-media thickness ${ }^{27}$. Hence, the present study suggests that there is a continuum in the association of E2 with cardiovascular risk and low grade inflammation in men, starting from childhood to adulthood. And in elderly men, where testosterone levels decline, relative abundance of E2 increases the cardiovascular risk. We obviously need future studies to test the role of altered sex hormone levels as a vascular risk factor in children and adolescents.

\section{Study limitations}

Our study was cross sectional and has several limitations. Fat distribution, insulin resistance, hormone levels, subclinical inflammation and lifestyle factors follow non-linear trajectories with complex interactions during the age range of the study participants (8-17 years). The study hypothesis 'androgen/estrogen 
balance contributes to the changes in hs-CRP levels among children and adolescents' cannot be addressed using a cross-sectional study. Furthermore, the study combines children and adolescents of all Tanner stages (which were not determined in the study) and merely adjusts for age as potential confounder. Given the age range that we studied, our findings most likely reflect the effects of profound changes in endogenous sex hormone levels with puberty.

Prospective studies are needed to assess temporal changes in the relation of hs-CRP with sex hormones in children and adolescents. To test the effects of diet and environmental factors, larger cohorts are needed. Methodologies and blood collection procedures may account for the differences. We used standardized procedures in an internationally accredited laboratory. Finally, insulin resistance was assessed by HOMA-IR, which a well-validated index in adults, but not extensively standardized in children and adolescents.

FEI levels associate with subclinical inflammation in male children. Future and prospective studies can confirm the role of sex hormones in the modulation of cardiometabolic risk and subclinical inflammation in children.

\section{REFERENCES}

1. Expert Panel on Integrated Guidelines for Cardiovascular Health and Risk Reduction in Children and Adolescents; National Heart, Lung, and Blood Institute. Expert panel on integrated guidelines for cardiovascular health and risk reduction in children and adolescents: summary report. Pediatrics 2011; 128: S213-256.

2. Agirbasli M, Agaoglu NB, Orak N, et al. Sex hormones, insulin resistance and high-density lipoprotein cholesterol levels in children. Horm Res Paediatr 2010; 73: 166-174.

3. Emerging Risk Factors Collaboration, Kaptoge S, Di Angelantonio E, et al. C-reactive protein, fibrinogen, and cardiovascular disease prediction. N Engl J Med 2012; 367: 1310-1320.

4. Agirbasli M, Tanrikulu A, Acar Sevim B, Azizy M, Bekiroglu N. Total cholesterol-to-high-density lipoprotein cholesterol ratio predicts high-sensitivity C-reactive protein levels in Turkish children. J Clin Lipidol 2015; 9: 195-200.

5. Berenson GS, Agirbasli M, Nguyen QM, Chen W, Srinivasan SR. Glycemic status, metabolic syndrome, and cardiovascular risk in children. Med Clin North Am 2011; 95: 409-417.

6. Hatipoglu N, Ozturk A, Mazicioglu MM, Kurtoglu S, Seyhan S, Lokoglu F. Waist circumference percentiles for 7- to 17-year-old Turkish children and adolescents. Eur J Pediatr 2008; 167: 383-389.
7. Cole TJ, Bellizzi MC, Flegal KM, Dietz WH. Establishing a standard definition for child overweight and obesity worldwide: international survey. BMJ 2000; 320: 12401243.

8. Selby C. Sex hormone binding globulin: origin, function and clinical significance. Ann Clin Biochem 1990; 27: 532-541.

9. Laakso M. How good a marker is insulin level for insulin resistance? Am J Epidemiol 1993; 137: 959965.

10. Kim J, Bhattacharjee R, Kheirandish-Gozal L, et al. Insulin sensitivity, serum lipids, and systemic inflammatory markers in school-aged obese and nonobese children. Int J Pediatr 2010; 2010: 846098.

11. Agirbasli M, Tanrikulu AM, Berenson GS. Metabolic syndrome: Bridging the gap from childhood to adulthood. Cardiovasc Ther 2016; 34: 30-36.

12. Karabouta Z, Papandreou D, Makedou A, Rousso I, Athanassiadou F. Associations of apolipoprotein A, high-sensitivity $\mathrm{C}$-reactive protein and fasting plasma insulin in obese children with and without family history of cardiovascular disease. J Clin Med Res 2016; 8: 431-436.

13. Agirbasli M, Agaoglu NB, Orak N, et al. Sex hormones and metabolic syndrome in children and adolescents. Metabolism 2009; 58: 1256-1262.

14. Oettel M. Is there a role for estrogens in the maintenance of men's health? Aging Male 2002; 5: 248-257.

15. Sørensen K, Aksglaede L, Munch-Andersen T, et al. Sex hormone-binding globulin levels predict insulin sensitivity, disposition index, and cardiovascular risk during puberty. Diabetes Care 2009; 32: 909-914.

16. Emerging Risk Factors Collaboration, Kaptoge S, Di Angelantonio E, et al. C-reactive protein concentration and risk of coronary heart disease, stroke, and mortality: an individual participant meta-analysis. Lancet 2010; 375: 132-140.

17. Swerdloff RS, Odell WD. Hormonal mechanisms in the onset of puberty. Postgrad Med J 1975; 51: 200-208.

18. Sharpe RM. Reproductive biology: do males rely on female hormones? Nature 1997; 390: 447-448.

19. Faustini-Fustini M, Rochira V, Carani C. Oestrogen deficiency in men: where are we today? Eur J Endocrinol 1999; 140: 111-129.

20. Veldhuis JD, Sowers JR, Rogol AD, Klein FA, Miller N, Dufau ML. Pathophysiology of male hypogonadism associated with endogenous hyperestrogenism. Evidence for dual defects in the gonadal axis. N Engl J Med 1985; 312: 1371-1375.

21. Onat A, Can G, Hergenç G. Serum C-reactive protein is an independent risk factor predicting cardiovascular risk. Metabolism 2008; 57: 207-214.

22. Hergenç G, Schulte H, Assmann G, von Eckardstein A. Associations of obesity markers, insulin, and sex hormones with HDL-cholesterol levels in Turkish and German individuals. Atherosclerosis 1999; 145: $147-$ 156. 
23. Canoy D, Barber TM, Pouta A, et al. Serum sex hormone-binding globulin and testosterone in relation to cardiovascular disease risk factors in young men: a population-based study. Eur J Endocrinol 2014; 170: 863-872.

24. Kupelian V, Chiu GR, Araujo AB, Williams RE, Clark RV, McKinlay JB. Association of sex hormones and C-reactive protein levels in men. Clin Endocrinol (Oxf) 2010; 72: 527-533.

25. Tsilidis KK, Rohrmann S, McGlynn KA, et al. Association between endogenous sex steroid hormones and inflammatory biomarkers in US men. Andrology 2013; 1: 919-928.
26. Tomaszewski M, Charchar FJ, Maric C, et al. Association between lipid profile and circulating concentrations of estrogens in young men. Atherosclerosis 2009; 203: 257-262.

27. Philips GB, Castelli WP, Abbott RD, McNamara PM Association of hyperestrogenemia and coronary heart disease in men in the Framingham cohort. Am J Med 1983; 74: 863-869. 